\title{
State Pod Automatism as Art Therapy.
}

Dr Bheemaiah, Anil Kumar, A.B Seattle W.A 98125

miyawaki@yopmail.com

\begin{abstract}
:
Formal art therapy is defined in a definition of the formal elements in art and metrics on structure, contour, variation and balance, leading to adaptability and measures of dynamism and thought as an emergence in the art form, leading to a viable diagnosis. In this paper we examine formal art analysis in art therapy and describe several metrics based on common data mining algorithms.

Keywords: Formal Art Therapy, Autopilot, Mindfulness, Wilderness Therapy, structure, variation, segmentation, objects in images, dynamism, balance, color histograms, $\mathrm{k}$ means, data mining.
\end{abstract}

What:

In understanding the Autopilot, we use art therapy in the form of the state pod automatism,in this coding , the formal elements consist of a flexible line composition with five or more anchor points to create pods, which are painted in a spectrum of colors using a pixel brush, either as oil or water colors, in as many colors and hues as possible, the transitions between the pods, represent the nature of the inertial, the reason for dukka.(Contributors to Wikimedia projects 2001)

We present data mining algorithms for structure and color, and contour based metrics for assisting a prognosis and art therapy formulation.

How:

Three examples are presented and analyzed using k-means algorithms to create statistics for colors, color clusters and pixels, with a database lookup of matching words as a meta description of the images. We present the analysis, and propose contour and structure mining tools for generating metrics.(Contributors to Wikimedia projects 2005)

\section{Introduction.}

Brain scans have revealed that when your mind wanders, it switches into "autopilot" mode, enabling you to carry on doing tasks quickly, accurately and without conscious thought. ... This research provided the first evidence that our brains are active even when we aren't consciously putting our minds to work.(Ings n.d.)
("Website" n.d., "Website" n.d., "Website" n.d.) A search in medical literature, in reference to wellness literature, hints many recipes to mental wellness and a clear separation from the habits of the autopilot, mindfulness being the first path, naturalness, wilderness therapy, and many more.

Autopilot thinking stems from an interplay of the waking state, in association with other 
states of living, predominantly the subconscious and dream, and higher states of consciousness. In an intersection of these states, is the origins of the state pod automatism, an automatism of abstraction in the intersections of life as represented in abstract pods interconnecting the many states of being, expressed in formal elements in a simple functional coding. The elements of color and dynamism are born from the internal intuition of automatism. In this paper we explore the use of data mining in adding metrics to this technique and extensions to all other art therapy techniques.

The Major Art Therapy Techniques are:.
A.Bridge Drawing
B. Kinetic-Family-Drawing (KFD)
C. House-Tree-Person (HTP)/Kinetic
House-Tree-Person (K-H-T-P)
D. Lowenfeld Developmental Stages of Art
E. Person Picking an Apple from a Tree (PPAT)

F. Bird's Nest Drawing (BND)

G. Draw-A-Person-In-The-Rain (DAP-R, PIR)

H. Expressive Therapies Continuum (ETC)

I. Draw-A-Story (DAS)

J. Diagnostic Drawing Series (DDS)

K. Road Drawing

L. Administer a formal art therapy assessment

While abstractionism is the formless, most other forms of art gain from a contour analysis, a semantic segmentation and tagging, for the analysis of the patterns of thought and dynamism, emergent from the art for a diagnosis and therapy definitions.In this paper we consider the use of mindfulness and art therapy as part of wilderness therapy towards transitioning out of autopilot.

\section{Problem Definition.}

State Pod Automatism.

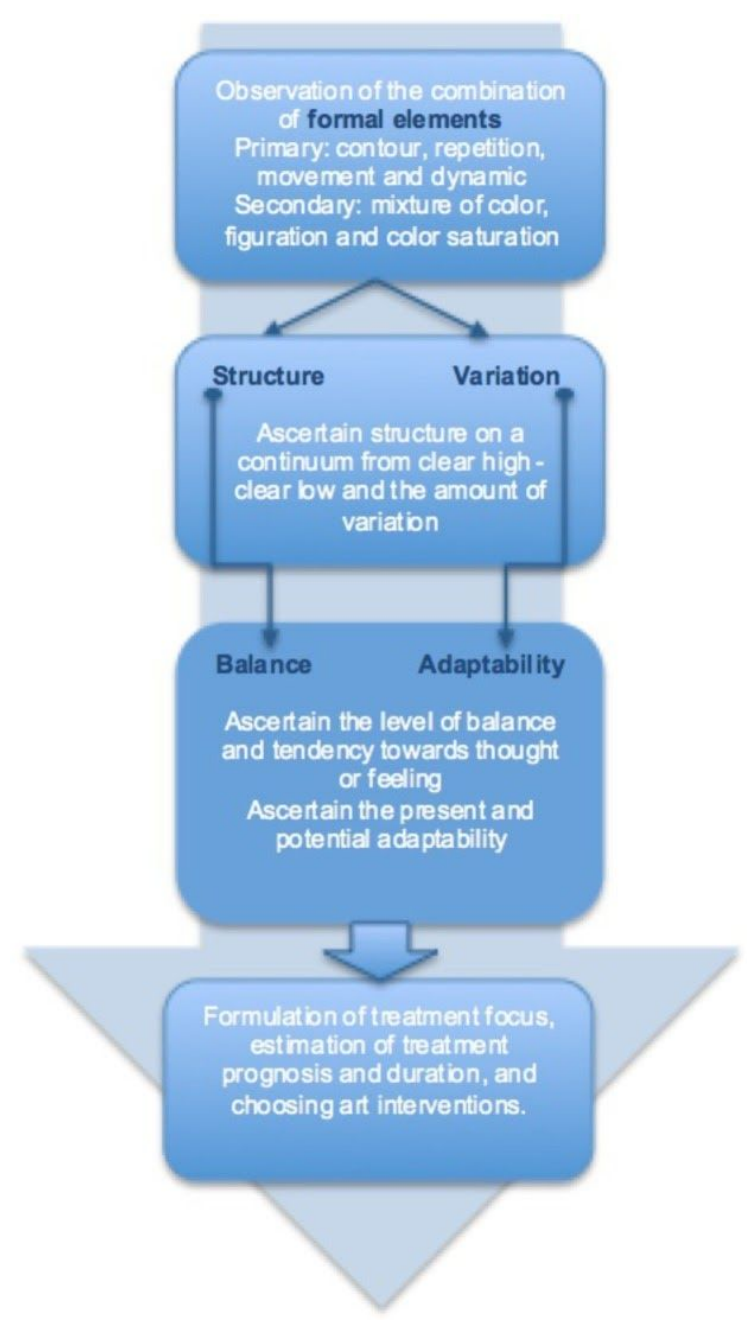

(Pénzes et al. 2018) is an analysis of the quantitative methods in formal art therapy, from structure and variation. In the above figure they summarize the flow in formal art therapy diagnosis. The Main tenets with possible data mining methods applicable to formal analysis are:

1. Color distribution and balance. metrics, color name and pixel count. color saturation metric. Color neighbourhood statistics. Segmentation of color boundary, and cluster neighbour coloring problem. 
2. Contour analysis, cartoonized feature map for analysis.

3. panoptic segmentation, using SLAM and segmentation for motion and dynamics detection, ontological API for motion and dynamic metaphors in feature space.

4. structure and variation, clear high to clear low on ontological API.

5. balance, in art metric, using the structure is to be defined with respect to colors and hues, as defined in a spatial statistical measure over the image.

6. adaptability metric, is to be defined over the image using variation defined over formal elements mapped to a feature space..

Data mining algorithms, for pixel and pixel cluster analysis.("Website" n.d.) are applicable to non form based abstractionism, where the pods clearly define the structure and the contours and a feature space is not clearly defined in ontologies. Hence both variation and structure, are defined over color, pixel and hue and saturation statistics, which can be browsed for the three example images using the links below, Art Therapy Image 001. Art Therapy Image 002. Art Therapy Image 003.
Analysis using metrics.
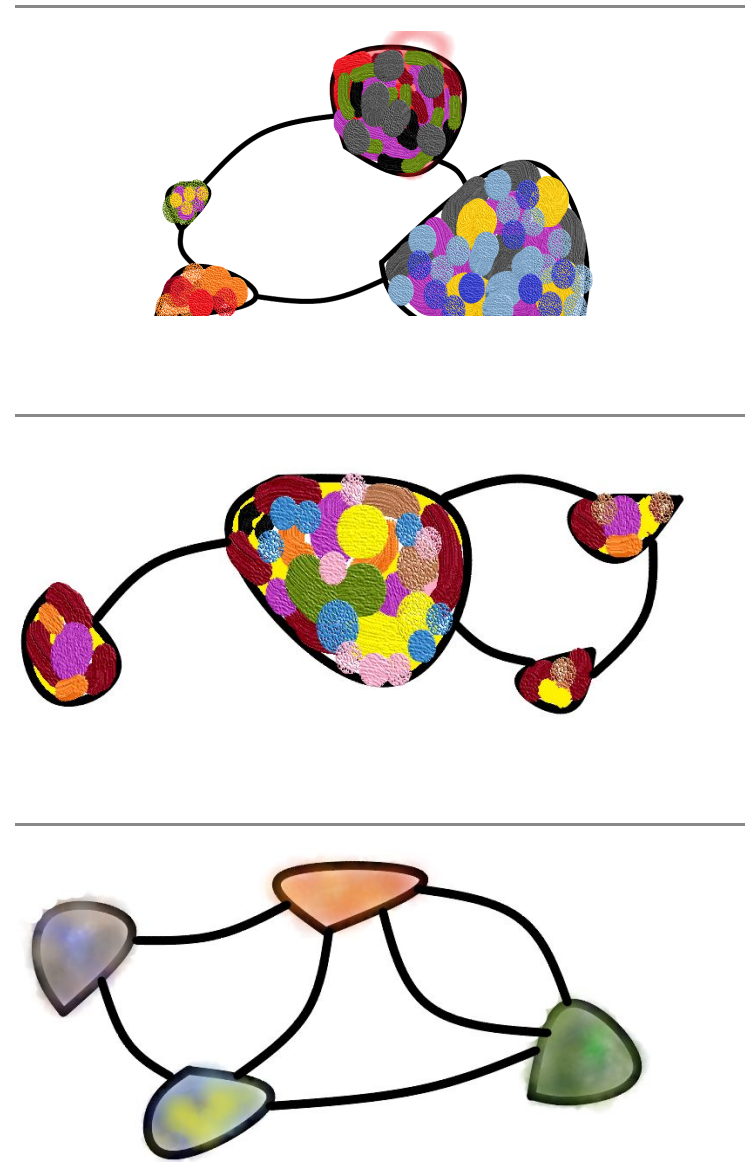

\section{Discussion.}

State Pod Automatism, leads directly to a practice of mindfulness based art therapy, In this paper, we have described a method of formal art assessment using data mining algorithms, such as K-Means and quantum clustering. While we avoid the design of a HL7 based CDS hook and expert system for formal analysis, the data mining algorithms provide metrics and numbers for diagnosis, 
enabling easy diagnosis from abstract art, rendered in the state and pod abstraction as formal elements.

The emergence of balance, variation and dynamism, are retained as subjective perceptions, rather than formulated as rule based heuristics.

The three links lead to a cluster and color statistic link , to a service authored by Martin Krzywinski I mkweb.bcgsc.ca, which, amongst several image data mining software including the Weka tool kit ("Weka 3 - Data Mining with Open Source Machine Learning Software in Java" n.d.)enables the creation of diagnostics from art images.

\section{Future Work.}

Future work brings more data mining and metrics to the world of formal elements in art, and the use of statistical and quantum methods, for metrics of variation and adaptability and dynamism. Contour analysis, feature spaces are directly amenable to a point cloud transformation("Visualize Paintings in Feature Space: New in Wolfram Language 12 " n.d.), and the use of the machine learning toolkit in Wolfram for art analysis of formal elements.

\section{References.}

Contributors to Wikimedia projects. 2001. "Duhkha," October.
https://en.wikipedia.org/wiki/Du\%E1\%B8 $\%$ A5kha.

2005. "K-Means Clustering," May. https://en.wikipedia.org/wiki/K-means_clus tering.

Ings, Simon. n.d. "Bloody Exhibition Is Only for the Brave and the Bold | New Scientist." New Scientist. Accessed May 30, 2020. https://www.newscientist.com/article/21510 03-bloody-exhibition-is-only-for-the-braveand-the-bold/.

Pénzes, Ingrid, Susan van Hooren, Ditty Dokter, and Giel Hutschemaekers. 2018. "How Art Therapists Observe Mental Health Using Formal Elements in Art Products: Structure and Variation as Indicators for Balance and Adaptability." Frontiers in Psychology 9. https://doi.org/10.3389/fpsyg.2018.01611.

"Visualize Paintings in Feature Space : New in Wolfram Language 12." n.d. Accessed June 3, 2020 .

https://www.wolfram.com/language/12/hig h-level-machine-learning/visualize-painting s-in-feature-space.html?product=language.

“Website.” n.d. Accessed May 30, 2020a. Jordan, R. (2015, June 30). Stanford researchers find mental health prescription: Nature. Retrieved from https://news.stanford.edu/2015/06/30/hikin g-mental-health-063015/.

- n.d. Accessed May 30, 2020b. Gilkey, R., \& Kilts, C. (2007). Cognitive Fitness. Retrieved from https://hbr.org/2007/11/cognitive-fitness.

- n.d. Accessed May 30, 2020c. Sage Publications. (2008, February 19). Does Socializing Make Us Smarter? Retrieved from https://www.sciencedaily.com/releases/200 8/02/080215135707.htm.

—. n.d. Accessed May 30, 2020d. https://mkweb.bcgsc.ca/color-summarizer/? home.

"Weka 3 - Data Mining with Open Source Machine Learning Software in Java." n.d. Accessed June 3, 2020. https://www.cs.waikato.ac.nz/ml/weka/. 
